\title{
3D strain mapping inside materials by microstructural tracking in tomographic volumes
}

\author{
H. Toda ${ }^{1}$, M. Kobayashi ${ }^{1}$, K. Uesugi ${ }^{2}$, D. S. Wilkinson ${ }^{3}$ \\ \& T. Kobayashi ${ }^{1}$ \\ ${ }^{1}$ Department of Production Systems Engineering, \\ Toyohashi University of Technology, Japan \\ ${ }^{2}$ Japan Synchrotron Radiation Research Institute, Japan \\ ${ }^{3}$ Department of Materials Science and Engineering, \\ McMaster University, Canada
}

\begin{abstract}
$\mathrm{X}$-ray absorption microtomography has been employed to trace the physical displacement of internal microstructural features in order to obtain local strain distribution inside materials. The number of microstructural features visible by means of state-of-the-art synchrotron radiation microtomography sometimes reaches into the tens of thousands or more in ordinary structural materials. It implies that high-density strain mapping is enabled if such large-scale tracking is accurately performed. The present paper describes a method to accurately track microstructural features by utilising the information on the size, shape and gravity centre of microstructural features together with the spring model particle tracking algorithm and exploratory registration using macroscopic deformation pattern. A model material which contains artificially introduced micro-pores has been prepared and used for the investigation. It has been clarified that almost perfect tracking is realised if the procedure is adequately applied to 3D image data sets. 3D internal strain mapping is also demonstrated and correlated to the localised ductile fracture of the model material.

Keywords: microtomography, tracking, three dimension, strain mapping, microstructure, spring model, ductile fracture, image analysis.
\end{abstract}




\section{Introduction}

As the resolution level of the synchrotron radiation microtomography is improved $[1,2]$, it has gradually come into use in various materials science and engineering fields in this decade. Especially, an in-situ observation utilising a specially designed rig has been proved to be a highly effective way of directly assessing various physical phenomena via the quantification of internal physical quantities three-dimensionally.

For example, Maire et al [3] investigated 2-D strain distribution in a $\mathrm{SiC}_{\mathrm{f}} / \mathrm{Ti}$ composite combining the X-ray synchrotron radiography and X-ray diffraction. 3D internal strain measurement was demonstrated by Bay [4] for a trabecular bone. Random surface speckle is automatically recognized in the technique as a basis for correlation. Other approaches have been reported recently for plastic displacement field visualisation by Nielsen et al [5], local crack opening displacement measurement by Toda et al [6] and fatigue crack driving force determination by Toda et al [7]. In these examples, microstructural features, such as particles or micro-pores were extracted by applying a fixed threshold value for each tomographic data thereby gravity centre of each feature is determined. Matching identical microstructural features in different datasets is performed semi-automatically by applying simple matching criterion such as physical displacement and/or the shape and the size of each microstructural feature. Some matching errors were inevitably included in the results due to the irregular trajectory caused by local deformation or resemblance in the shape and size of features, by which laborious manual corrections were subsequently obliged to delete them almost manually [5,7]. Another approach to derive internal strain distribution is the digital image correlation technique. Concerning the high resolution microtomography images in which a secondary phase has a sharp interface and different X-ray absorption with a matrix, the former seems to be clearly advantageous. Particles and micro-pores embedded in a metal would be such examples. On the other hand, the latter would be potentially effective in biological objects.

This paper reports how the accuracy of tracking numbers of microstructural features is ensured by applying several different procedures. Experimental verification of accuracy is performed by producing a model material in which micro-pores of known size and spatial distribution is artificially introduced.

\section{Experimental and image analysis procedures}

\subsection{Image analysis}

\subsubsection{Preliminary image processing}

The tomographic volumes are first processed to extract microstructural features. The features are segmented using a fixed threshold value and then converted into a binary image with either gray values of 0 (background) or 1 (object). A labelling algorithm is subsequently applied in order to distinguish features. Geometrical information of each microstructural feature, i.e. surface area, 
volume and gravity centre are calculated utilizing the Marching Cube algorithm that gives pentagonal faceted isointensity surface [8]. Before tracking each marker in a set of tomographic volumes, registration among volumes is performed as a first step to compensate translational and rotational shifts relative to each other. The registration is executed as follows; first, eight sets of tracking marker pairs, which can be readily distinguished from surrounding markers, are selected manually in each tomographic volume. A home-made software that can output marker locations is used for it. Second, a transformation matrix is calculated so that the sum of differences in distance between corresponding pairs is minimised. It is applied to all the makers to superpose a chronologically late volume onto a chronologically-advanced volume.

\subsubsection{Base tracking procedure using matching probability parameter}

As is easily supposed, deformation or deterioration of a real material does not allow correct correlation if only the above simple registration is applied. A matching probability parameter (MPP), $M_{p}$, is introduced to extract tracking errors as well as correctly matched pairs. This is fundamentally two-frame particle tracking procedure [9]. Index numbers, $i$ and $j$, denote feature number which is assigned in the labelling process.

$$
M_{P}=\alpha L_{i, j}+\beta S_{i, j}+\gamma V_{i, j}
$$

Here $L_{i, j}, S_{i, j}$ and $V_{i, j}$ are location, surface area and volume parameters, respectively. $\alpha, \beta$ and $\gamma$ are weights, where $\alpha+\beta+\gamma=1$. Firstly one microstructural feature is picked up from a quantified dataset, and then $M_{p}$ values for all markers in another corresponding dataset are calculated using eqn (1). If the maximum $M_{p}$ value is larger than a pre-determined first threshold value, $M_{p}^{\text {thl }}$, and a difference between the maximum and the second largest values is larger than a pre-determined second threshold value, $M_{p}{ }^{\text {th } 2}$, then the marker pair with the largest $M_{p}$ value is labeled to be tracked (Hereafter it is called "tracked marker".). If the difference is less than $M_{p}^{t h 2}$, then the marker pair is held in a waiting list (Hereafter it is called "pended marker".). If no marker satisfies the first criterion on $M_{p}^{\text {thl }}$, the marker is not used for the tracking purpose (Hereafter it is called "rejected marker".). By repeating the above operation for all the markers in the first dataset, the initial tracking procedure is terminated. In this study, the $M_{p}^{\text {thl }}$ and $M_{p}^{\text {th2 }}$ values are set to be 0.8 and 0.1 , respectively after preliminary investigation. The definition of $L_{i, j}$ is as follows:

$$
L_{i, j}=\left\{\begin{array}{cl}
\left(r_{s}-l_{i, j}\right) / r_{s} & , l_{i, j} \leq r_{s} \\
0 & , l_{i, j}>r_{s}
\end{array}\right.
$$

where $l_{i, j}$ is a distance between the marker with index $i$ in the first dataset and the marker with index $j$ in the second dataset, $r_{s}$ is the radius of a search space in which corresponding marker is searched. Therefore $L_{i, j}$ increases when a marker suspected to be identical is located close to the relevant marker, and becomes 0 outside the search space. $S_{i, j}$ and $V_{i, j}$ are also defined in a similar way. 


\subsubsection{Macroscopic trajectory prediction}

Two kinds of additional tracking procedures are used together with the abovementioned procedure. One is a macroscopic trajectory prediction (MTP) on the basis of known macroscopic deformation pattern. The method adopted here is quite simple. Provided that applied strain distributes homogeneously throughout a material, centre of gravity coordinate of each marker in the image dataset of a deformed sample is linearly interpolated before the two-frame tracking algorithm described in the section 2.1.2 is applied. In this study, the model sample was monotonously tensioned, so the image dataset has been just extended in the longitudinal direction and contracted in the vertical directions assuming Poisson's ratio of 0.5 .

\subsubsection{Spring model tracking algorithm}

Another tracking algorithm, which is called the modified spring model algorithm (SMA) [10] is applied for markers classified as "Pended". The spring model tracking algorithm is one of the particle image velocity methods to measure the velocity of flows which has been utilised in the field of fluid mechanics. The schematic illustration of this method is shown in Fig. 1. This method identifies particle pairs by searching the smallest total spring force among particle clusters, in which particles are connected by imaginary springs each other. The method would be effective for locally inhomogeneous deformation, because the model can cope with apparently random deviation of relative position among the markers.

\subsubsection{Post processing and final assessment}

Finally the assessment of tracking results is performed by calculating the ratio of success tracking, $\eta$, which is the number of correctly tracked markers normalised by that of all the markers. The correctness is checked using a database in which the trajectory of each marker has been examined in advance and recorded.

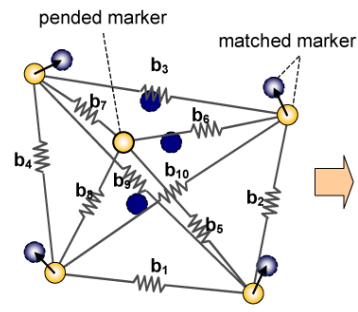

Before deformation

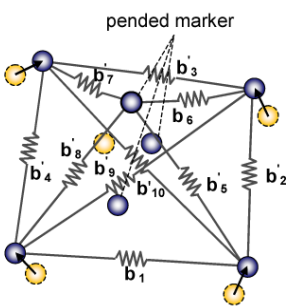

After deformation

Figure 1: Schematic illustration of the spring model for matching pended markers. Provided that four pended markers are observed in the second image. One of which is suspected to be identical to a single marker observed before deformation.

Tetrahedrons, whose vertex is occupied by markers, are generated by the Delaunay tessellation technique [12]. Normal and shear strains are calculated 
from the deformation of the tetrahedron assuming a linear displacement field inside it. Local strain distribution is demonstrated as a form of cross-sectional or $3 \mathrm{D}$ colour contour maps.

\subsection{Model material}

A model sample used to examine the performance of the series of the procedures is a dispersion-strengthened copper in which micro-pores of uniform size have been introduced artificially. The material consists of four sheets containing holes of $15 \mu \mathrm{m}$ in diameter all the way through each sheet and five hole-free sheets, which were sandwiched alternately and then diffusion-bonded. The thickness values of the sheets are 15,30 and $50 \mu \mathrm{m}$ for the drilled sheets and the inner and outer hole-free sheets, respectively. In total 188 pores were introduced in the model sample. Tensile test specimens with a cross-section of $250 \times 300 \mu \mathrm{m}$ were cut out from the joined plate.

\subsection{Tomographic observation of deformation and fracture}

High-resolution tomographic experiments were performed using a monochromatic X-ray beam having a photon energy of $35 \mathrm{keV}$ at beamline BL20XU in the synchrotron radiation facility SPring-8. The tensile load was applied by successively increasing displacement using a test rig specially designed for this purpose [11]. Four CT scans were performed for a specimen; one prior to loading and the other three at different loading states. An isotropic voxel with a cutting edge of $0.474 \mu \mathrm{m}$ was achieved using the present set up. Each voxel has a 16-bit gray-scale depth, which corresponds to linear absorption coefficient (LAC) value. To reduce the size of image sets, 16-bit LAC values ranging 0 to 80 was linearly mapped to a range between 0 and 255 in an 8-bit gray-scale. The other details of the imaging procedure are available elsewhere [11].

\section{Evaluation of results}

Figure 2 shows a 3D reconstructed image captured at the final loading step which is just before the final rupture. All the micro-pores has been cylindrical and of uniform size before loading. Randomly distributed micro-pores lying on the four layers appear to overlap in the figure. The micro-pores have been gradually elongated and then necking region is observed as is shown in fig. 2 . Damage evolution is observed in the figure where some of the micro-pores located in the necked region are linked up with each other to be a coarse cracklike damage. Macroscopic applied strain was measured using a couple of markers located at almost the both ends of the field of view shown in the figure. The applied strain ranged between 3.5 and $22.1 \%$. Closer inspection revealed that interlayer delamination and its interaction with micro-pores.

Changes in the ratio of tracked, pended, rejected and correctly tracked markers for all the markers, $m$ with the radius of a search space, $r_{s}$, are shown in fig. 3 where only the basic MPP method is applied. Two examples for different 
applied strain difference, $\Delta$, between the two consecutive images (3.5 and $16.3 \%$ ) are included in the figure. Apparently $16.3 \%$ strain difference is too large for an ordinary in-situ experiment since the common fracture strain of engineering materials: several ten $\%$ divided by 10 observation stages falls to several\% strain difference. Therefore it is an unrealistic extreme case to test the potential of the procedure. It can be seen that the number of tracked markers has a maxima at $r_{s}=71.1$ and $106.7 \mu \mathrm{m}$ for $\Delta$ of 3.5 and $16.3 \%$, respectively. Although the available marker points vary with $r_{s}$, perfect matching was obtained for $\Delta$ of $3.5 \%$, while it is fairly low (29.0\% at maximum) for $\Delta$ of $16.3 \%$.

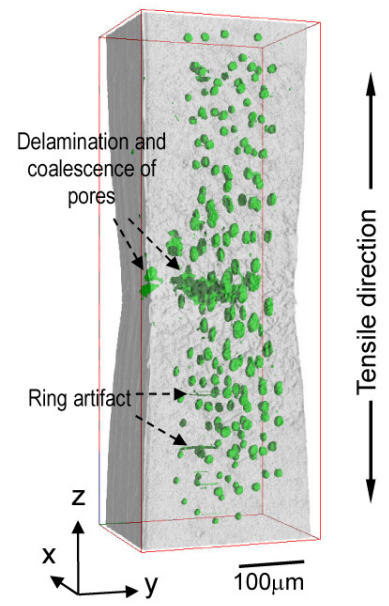

Figure 2: A reconstructed 3D image acquired at the fourth loading step, representing inner pore (in green) and damage evolution in the necked region.
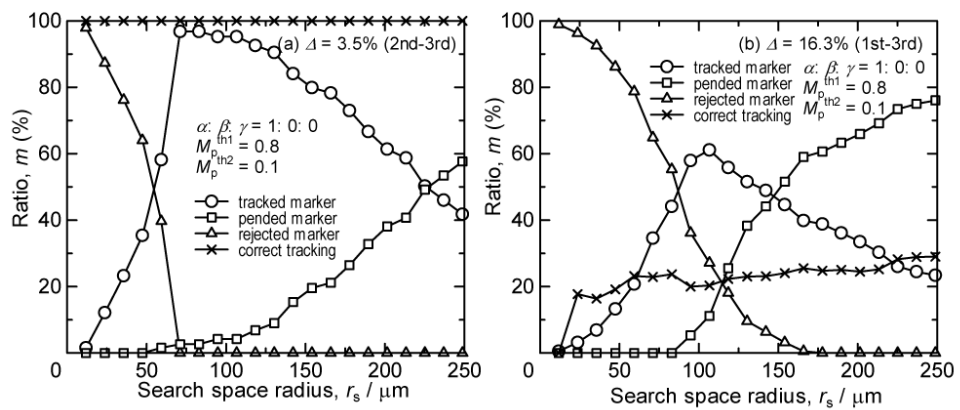

Figure 3: Changes in the ratio of tracked, pended, rejected and correctly matched markers with increasing the size in search space, $r_{\mathrm{s}}$, for the strain differences of (a) 3.5\% and (b) 16.3\%. 


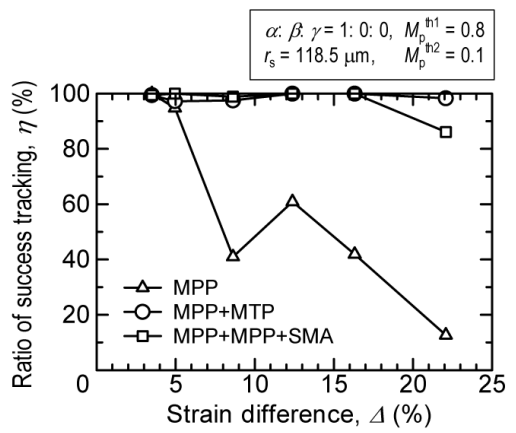

Figure 4: Changes in the ratio of success tracking with applied strain. The matching probability parameter (MPP) is used for all, while the macroscopic trajectory prediction (MTP) and/or the spring model algorithm (SMA) are added in the remaining two trials.

The feasibility of the additional two tracking algorithm (MTP and SMA) can be seen in fig. 4. As shown in fig. 3, the ratio of success tracking decreases with macroscopic applied strain if only the basic MPP method is applied. The application of all three algorithm provides satisfactorily high success ratio below the applied strain of $16.3 \%$, while the application of SMA causes the deterioration in the ratio for a very large $\Delta$. This deterioration has little influence on practical measurement because $\Delta$ higher than $10 \%$ is practically unrealistic as described above. Figure 5 also shows the effects of search space. If only the MPP method is applied as shown in fig. 5(a), the ratio of success tracking is critical to the search space. This implies that considerable preliminary trial is needed to find out the optimum parameter, which seems to be quite impractical. When the MTP method is, however, added as shown in fig. 5(b), the ratio of success tracking ranges between 94.2 and $100 \%$ for $r_{s}$ above $23.7 \mu \mathrm{m}$. Therefore unless otherwise parameters are irrelevantly small, the accuracy of the method is quite insensitive to the parameter setting, which seems to be beneficial from a practical point of view.

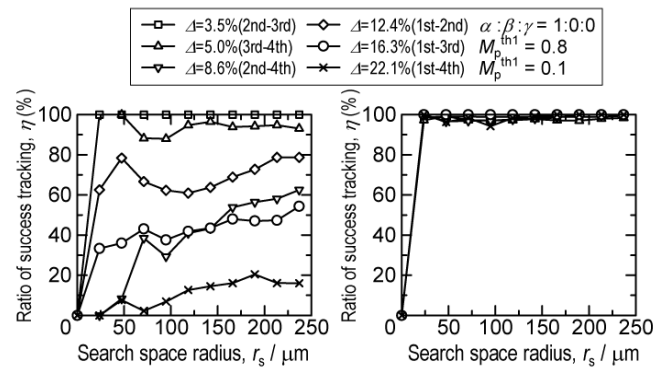

(a) MPP

(b) MPP+MTP

Figure 5: Ratio of success tracking against the size of search space, $r_{s}$. The matching probability parameter (MPP) is used in (a) and the macroscopic trajectory prediction (MTP) is added in (b). 


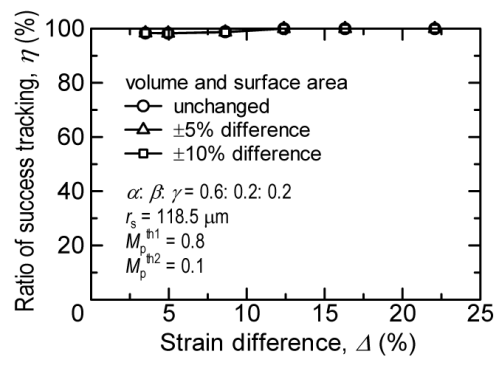

Figure 6: Ratio of success tracking for the tracking in consideration of volume parameter, $M_{\mathrm{v}}$, and surface area parameter, $M_{\mathrm{s}}$. Volume and surface area of each marker are intentionally varied with predetermined variation ranges. The other conditions are similar to fig. 4.

Figure 6 also demonstrates the effects of a likely supposition that the volume and surface area of each particle are different between two consecutive images. This is most likely when micro-pores grow under an applied loading. Partial dissolution or growth of a secondary phase during a secondary process such as a heat treatment also brings about the similar situation. The application of the MTP procedure provides almost perfect tracking even if such geometrical disturbance occurs.

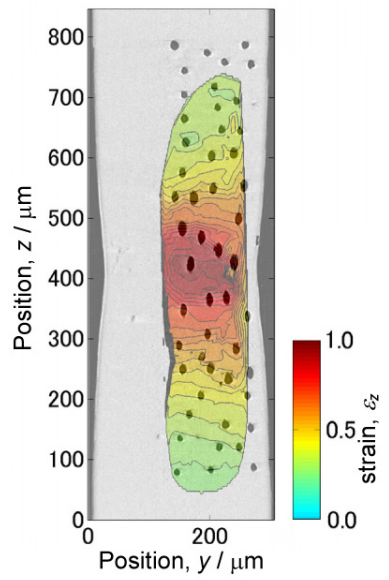

(a)

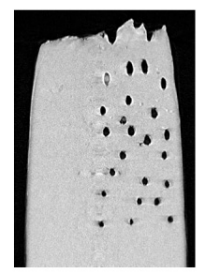

(b)

Figure 7: Comparison of cross-sectional representation of axial strain, $\varepsilon_{\mathrm{Z}}$, with a corresponding tomographic slice. Strain concentration along obliquely aligned pores and final fracture through them are clearly demonstrated.

It is concluded that the combination of the three tracking algorithms may lead to reliable and robust microstructural tracking, relatively independent of the 
parameter setting conditions: such stability may be seen to enable automatic accurate strain mapping when a suitable population of particles is identified, especially in the case of aluminium alloys which inherently contain small but closely interspersed intermetallic particles and micro-pores.

\section{Comparison with in-situ visualisation}

3D strain distribution and its change with applied loading have been successfully visualised utilising the present procedure. Fig. 7 is one of such examples. Although 3D display is possible using the method described in the section 2.1.5, strain distribution is shown in the figure on a 2-D virtual cross-section to show positional relationship between the aligned micro-pores and calculated localised strain directly. Fig. 7(a) clearly demonstrates that the upper four micro-pores lying along a downward-sloping line and the lower three micro-pores lying along a somewhat upward line cause strain localisation within the area surrounded by the micro-pores. A virtual slice on the identical cross-section shown in fig. 7(b) exhibits a final crack path on fracture passing through the upper pores. Overall, the high resolution in-situ synchrotron X-ray microtomography offers a highly effective way of visualising internal strain distribution in 3D which might give us a direct solution for the interpretation of realistic physical phenomenon.

\section{Summary}

A tracking procedure for microstructural features contained in the high resolution in-situ synchrotron X-ray microtomography image has been developed in order to measure 3D local strain distribution within a deforming material in highdensity. Especially it is crucial to raise its accuracy. The developed tracking method consists of the matching probability parameter, macroscopic trajectory prediction and spring model tracking algorithms. By combining these three algorithms, the robust procedure for internal strain visualisation is realised, being appropriate for automatic high-density mapping in the case of practical materials which inherently contain small and closely interspersed intermetallic particles or micro-pores. The tracking results were relatively independent of the parameter setting conditions and such stability may be crucial from practical point of view.

\section{Acknowledgements}

NEDO has been supported this work as Collaborative Research of Production and Fabrication Technology Development of Aluminum useful for Automobile Light-weighting. The supports of the Grant-in-aid for Scientific Research from JSPS through subject No. 17360340, JASRI through proposal number 2005A0066-NM-np, the Tatematsu Foundation and the Light Metal Educational Foundation are also gratefully acknowledged. 


\section{References}

[1] Cloetens, P., Pateyron-Salome', M., Buffie're, J.-Y., Peix, G., Baruchel, J., Peyrin, F., Schlenker, M., Observation of microstructure and damage in materials by phase sensitive radiography and tomography. J. Appl. Phys., 81(9), pp. 5878-5886, 1997.

[2] Toda, H., Uesugi, K., Takeuchi, A., Minami, K., Kobayashi, M. \& Kobayashi, T., Three-dimensional observation of nanoscopic precipitates in an aluminium alloy by microtomography with Fresnel zone plate optics. Applied Physics Letter, 89(14), pp. 143112, 2006.

[3] Maire, E., Owen, A., Buffiere, J.-Y., Withers, P.J., A synchrotron X-ray study of a $\mathrm{Ti} / \mathrm{SiCf}$ composite during in situ straining. Acta Materialia. 49, pp. 153-163, 2001.

[4] Bay, B.K., Texture correlation: A method for the measurement of detailed strain distributions within trabecular bone. Journal of Orthopaedic Research, 13(2), pp. 258-267, 1999.

[5] Nielsen, S.F., Poulsen, H.F., Beckmann, F., Thorning, C., Wert, J.A., Measurements of plastic displacement gradient components in three dimensions using marker particles and synchrotron X-ray absorption microtomography. Acta Materialia, 51(9), pp. 2407-2415, 2003.

[6] Toda, H., Sinclair, I., Buffière, J.-Y., Maire, E., Connolley, T., Joyce, M., Khor, K.H., Gregson, P.J., Assessment of fatigue crack closure phenomenon in damage tolerant aluminium alloy by in-situ highresolution synchrotron X-ray microtomography. Philosophical Magazine, A83, pp. 2429-2448, 2003.

[7] Toda, H. Sinclair, I., Buffière, J.-Y., Maire, E., Khor, K.H., Gregson, P. and Kobayashi, T., A 3D measurement procedure for internal local crack driving forces via synchrotron X-ray microtomography. Acta Materialia. 52(5), pp. 1305-1317, 2004.

[8] Lohmann, G., Volumetric Image Analysis, John Wiley \& Sons Ltd: Chichester, pp. 183-186, 1998.

[9] Kim, H.-B., Lee, S.-J., Performance improvement of two-frame particle tracking velocimetry using a hybrid adaptive scheme, Measurement Science \& Technology, 13(4), pp. 573-582, 2002.

[10] Okamoto, K., Hassan, Y.A., Schmidt, W.D, New tracking algorithm for particle image velocimetry, Experimental Fluids, 19(5), pp. 324-347, 1995.

[11] Toda, H., Ohgaki, T., Uesugi, K., Kobayashi, M., Kuroda, N., Kobayashi, T., Niinomi, M., Akahori, T., Makii, K. and Aruga, Y., Quantitative assessment of microstructure and its effects on compression behaviour of aluminium foams via high-resolution synchrotron X-ray tomography, Metallurgical and Materials Transactions. A, 37A(4), pp. 1211-1220, 2006.

[12] Barber, C.B., Dobkin, D.P. and Huhdanpaa, H.T., The Quickhull Algorithm for Convex Hulls, ACM Transaction on Mathematical Software, 22(4), pp.469-483, 1996. 